\title{
Analysis of Some Results on Complete Fuzzy Metric Spaces and Separable Fuzzy Metric Spaces
}

\author{
Dipen Saikia
}

Research Scholar, Department of Mathematics, CMJ university, Meghalaya

\begin{abstract}
In this paper we present an analogue result in the context of fuzzy metric spaces. Again we show that a product of two complete fuzzy metric spaces is also a complete fuzzy metric space and subspace of a separable fuzzy metric space is also separable.
\end{abstract}

Keywords: Metric Space, Fuzzy metric space, complete fuzzy metric space, Fuzzy diameter zero, Separability, uniform convergence in fuzzy metric space.

\section{Introduction}

The concept of fuzzy sets and fuzzy logic was introduced by Professor Lofti A Zadeh in 1965. The success of research in fuzzy sets and fuzzy logic has been demonstrated in a variety of fields, such as artificial intelligence, computer science, control engineering, computer applications, robotics and many more. In this chapter we adopt the notion of fuzzy metric space due to George and Veeramani [1]which is modification of the notion of fuzzy metric space as studied by Kramosil and Michalek[2]. The notion of fuzzy metric space by George and Veeramani has many advantages in analysis as many notions and results from classical metric spaces can be extended and generalized to the setting of fuzzy metric spaces, for instance: the notion of completedness, completion of spaces as well as extension of maps.

This chapter is based on the work due by A George and P Veeramani [1]. We shall recall the definition of a fuzzy metric space which was modified from [2] to obtain the Hausdorff topology on a fuzzy metric space. In this chapter we expand on the paper [1] by means of providing detailed examples, propositions, remarks and proofs of some results. Most of work presented in this section is well known and can be found in the literature, see[3],[4], [5], [6], [7] and [8].

\section{Prelimanaries:1.1}

Definition 1.1.1. Let $\left(X_{1}, d_{1}\right.$ and $\left(X_{2}, d_{2}\right)$ be metric space and let $x=\left(x_{1}, x_{2}\right)$ and $y=\left(y_{1}, y_{2}\right)$ be arbitrary points in the product $\quad X=X_{1} \times X_{2}$. Define $d(x, y)=\max \left\{d_{1}\left(x_{1}, y_{1}\right), d_{2}\left(x_{2}, y_{2}\right)\right\}$

Then $d(x, y)=\max \left\{d_{1}\left(x_{1}, y_{1}\right), d_{2}\left(x_{2}, y_{2}\right)\right\}$ is a metric on $X$ and $(X, d)$ called the product of the metric spaces $\left(X_{1}, d_{1}\right.$ and $\left(X_{2}, d_{2}\right)$.

Definition 1.1.2. A topological space $(X, \tau)$ is called first countable space iff it has a countable neighborhood base at each point.

Definition 1.1.3. Let $X$ be a topological space with topology $\tau$. A collection $B$ of subsets $X$ is called a base of $\tau$ if : (i) Each Member of $B$ is open in $X$, (ii) Each open subset of $X$ is the union of some collection of sets belonging to $B$.
Definition 1.1.4. A topological space is said to be second countable or is said to satisfy the second axiom of countability if the topology on the space can be generated by countable base.

Definition 1.1.5. Let $(X, d)$ be a metric space. If there is a countable dense subset in $(X, d)$ then, $(X, d)$ is said to be separable.

Proposition 1.1.1. Let $\left(X_{n}, d_{n}\right), n=1,2, \ldots \ldots \ldots$ be a metric spaces. Then $X=\prod_{n=1}^{\infty} X_{n}$ with the metric $d$ defined by $d(x, y)=\sum_{n=1}^{\infty} 2^{-n} d_{n}\left(x_{n}, y_{n}\right)$, Where $x=\left\{x_{n}\right\}$ and $y=\left\{y_{n}\right\}$ are in $X$, is a complete metric space iff each $\left(X_{n}, d_{n}\right), n=1,2, \ldots \ldots \ldots$ is complete.

Proposition 1.1.2. Let $(X, d)$ be a metric space and $A \subseteq X$. If $X$ is separable the $A$ with the induced metric is separable, too.

Theorem 1.1.1. Every nested sequence of nonempty closed sets with metric diameter zero has nonempty intersection.

Theorem 1.1.2. Every separable metric satisfies the second condition of countability.

Definition 1.1.6 A binary operation $*:[0,1] \times[0,1] \rightarrow$ $[0,1]$ is a continuous triangular norm(t-norm) if for all $a, b, c, d \in[0,1]$

M1. $a * 1=a$

M2. $a * b=b * a \quad$ (commutativity)

M3. $a * b \leq c * d$ whenever $a \leq c$ and $b \leq d$

M4. $a *(b * c)=(a * b) * c \quad$ (associativity)

Definition 1.1.7 The 3-tuple $(X, M, *)$ is said to be a fuzzy metric space, where $X$ is an arbitrary set, $*$ is continuous tnorm and $M$ is a fuzzy set on $X \times X \times[0, \infty)$ satisfying the following conditions:

M1. $M(x, y, 0)=0, \quad \forall x, y \in X$

M2. $M(x, y, t)=1$ iff $x=y, \forall t>0$ and $\forall x, y \in X$.

M3. $M(x, y, t)=M(y, x, t), \forall t>0$ and $\forall x, y \in X$.

M4. $\quad M(x, z, t+s) \geq M(x, y, t) * M(y, z, s), \forall s, t>$ 0 and $\forall x, y \in X$.

M5. $M(x, y):,[0, \infty) \rightarrow[0,1]$ is continuous, $\forall x, y \in X$.

Definition 1.1.8 The 3-tuple $(X, M, *)$ is said to be a fuzzy metric space where $X$ is an arbitrary set, $*$ is continuous t- 


\section{International Journal of Science and Research (IJSR) \\ ISSN (Online): 2319-7064}

Index Copernicus Value (2013): 6.14 | Impact Factor (2014): 5.611

norm and $M$ is a fuzzy set on $X \times X \times(0, \infty)$ satisfying the following conditions:

M1. $M(x, y, t)>0, \quad \forall x, y \in X$ and $\forall t>0$

M2. $M(x, y, t)=1$ iff $x=y, \forall t>0$ and $\forall x, y \in X$.

M3. $M(x, y, t)=M(y, x, t), \forall t>0$ and $\forall x, y \in X$.

M5. $\quad M(x, z, t+s) \geq M(x, y, t) * M(y, z, s), \forall s, t>$ 0 and $\forall x, y \in X$.

M6. $M(x, y, \cdot):(0, \infty) \rightarrow[0,1]$ is continuous , $\forall x, y \in X$.

\section{Results 1.2}

Now we present Proposition 1.1.1 to the fuzzy metric space setting.

Proposition 1.2.1 Let $\left(X_{1}, M_{1}, *\right)$ and $\left(X_{2}, M_{2}, *\right)$ be fuzzy metric spaces. We define $M\left(\left(x_{1}, x_{2}\right),\left(y_{1}, y_{2}\right), t\right)=$ $M_{1}\left(x_{1}, y_{1}, t\right) * M_{2}\left(x_{2}, y_{2}, t\right)$

Then $M$ is a complete fuzzy metric on $X_{1} \times X_{2}$ if and only if $\left(X_{1}, M_{1}, *\right)$ and $\left(X_{2}, M_{2}, *\right)$ are complete.

Proof: Suppose that $\left(X_{1}, M_{1}, *\right)$ and $\left(X_{2}, M_{2}, *\right)$ be fuzzy metric spaces.

Let $\left\{a_{n}\right\}$ be a Cauchy sequence in $X_{1} \times X_{2}$. Note that

and

$$
a_{n}=\left(x_{1}^{n}, x_{2}^{n}\right)
$$

$\left(x_{1}^{m}, x_{2}^{m}\right)$.

$$
a_{m}=
$$

Also, $M\left(a_{n}, a_{m}, t\right)$ converges to 1 . This implies that

$$
M\left(\left(x_{1}^{n}, x_{2}^{n}\right),\left(x_{1}^{m}, x_{2}^{m}\right), t\right)
$$

converges to 1 for each $t>0$. It follows that

$$
M_{1}\left(x_{1}^{n}, x_{1}^{m}, t\right) * M_{2}\left(x_{2}^{n}, x_{2}^{m}, t\right)
$$

converges to 1 for each $t>0$. Thus $M_{1}\left(x_{1}^{n}, x_{1}^{m}, t\right)$ converges to 1 and also $M_{2}\left(x_{2}^{n}, x_{2}^{m}, t\right)$ converges to 1 . Therefore $\left\{x_{1}^{n}\right\}$ is a Cauchy sequence in $\left(X_{1}, M_{1}, *\right)$ and $\left\{x_{2}^{n}\right\}$ is a Cauchy sequence in $\left(X_{2}, M_{2}, *\right)$. Since $\left(X_{1}, M_{1}, *\right)$ and $\left(X_{2}, M_{2}, *\right)$ are complete fuzzy metric spaces, there exists $x_{1} \in X_{1}$ and $x_{2} \in X_{2}$ such that $M_{1}\left(x_{1}^{n}, x_{1}, t\right)$ converges to 1 and $M_{2}\left(x_{2}^{n}, x_{2}, t\right)$ converges to 1 for each $t>0$. Let $a=\left(x_{1}, x_{2}\right)$. Then $a \in X_{1} \times X_{2}$. It follows that $M\left(a_{n}, a, t\right)$ converges to 1 for each $t>0$. This shows that $(X, M, *)$ is complete.

\section{Conversely,}

Suppose that $(X, M, *)$ is complete. We shall show that $\left(X, M_{1}, *\right)$ and $\left(X, M_{2}, *\right)$ are complete. Let $\left\{x_{1}^{n}\right\}$ and $\left\{x_{2}^{n}\right\}$ be Cauchy sequences in $\left(X, M_{1}, *\right)$ and $\left(X, M_{2}, *\right)$ respectively. Thus $M_{1}\left(x_{1}^{n}, x_{1}^{m}, t\right)$ converges to 1 and $M_{2}\left(x_{2}^{n}, x_{2}^{m}, t\right)$ converges to 1 for each $t>0$. It follows that

$$
M\left(x_{1}^{n}, x_{2}^{m}, t\right)=M_{1}\left(x_{1}^{n}, x_{1}^{m}, t\right) * M_{2}\left(x_{2}^{n}, x_{2}^{m}, t\right)
$$

Converges to 1 . Let $x^{n}=\left(x_{1}^{n}, x_{2}^{m}\right)$ in $X_{1} \times X_{2}$ for $n \geq 1$. Then $\left\{x^{n}\right\}$ is a Cauchy sequence in $X$. Since

$(X, M, *)$ is complete, there exists $x \in X_{1} \times X_{2}=X$ such that $M\left(x_{1}^{n}, x, t\right)$ converges to 1 . Since $x \in X_{1} \times X_{2}$, we may put $x=\left(x_{1}, x_{2}\right), x_{1} \in X_{1}$ and $x_{2} \in X_{2}$. Clearly, $M_{1}\left(x_{1}^{n}, x_{1}, t\right)$ converges to 1 and $M_{2}\left(x_{2}^{n}, x_{2}, t\right)$ converges to 1 . Hence $\left(X, M_{1}, *\right)$ and $\left(X, M_{2}, *\right)$ are complete. This completes the proof.

Definition 1.2.2. Let $(X, M, *)$ be a fuzzy metric space. A collection of sets $\left\{F_{n}\right\}_{n \in I}$ is said to have fuzzy diameter zero if for each pair $r, t>0,0<r<1$, there exists $n \in I$ such that

$$
M(x, y, t)>1-r
$$

for all $x, y \in F_{n}$.

Remark 1.2.3 A nonempty subset $F$ of a fuzzy metric space $X$ has fuzzy diameter zero if and only if $F$ is a singleton set, where $F=F_{n}$ for all $n \geq 1$.

We now generalize Theorem 1.1.1:

Theorem 1.2.4 A necessary and sufficient condition that a fuzzy metric space $(X, M, *)$ be complete is that every nested sequence of nonempty closed sets $\left\{F_{n}\right\}_{n=1}^{\infty}$ with fuzzy diameter zero has nonempty intersection.

Proof: First suppose that the given condition is satisfied. We claim that $(X, M, *)$ is complete. Let $\left\{x_{n}\right\}$ be a Cauchy sequence in $X$. Take

and

$$
A_{n}=\left\{x_{n}, x_{n+1}, x_{n+2}, \ldots\right\}
$$

$\overline{A_{n}}$,

$$
F_{n}=
$$

then we claim that $\left\{F_{n}\right\}$ has fuzzy diameter zero. For given $s, t>0,0<s<1$, we can find an $r \in(0,1)$, such that

$$
(1-r) *(1-r) *
$$

$(1-r)>(1-s)$

Since $\left\{x_{n}\right\}$ is a Cauchy sequence, for $r, t>0,0<r<1$, there exists $n_{0} \in N$ such that

for all $n, m \geq n_{0}$. Therefore

$$
M\left(x_{n}, x_{m}, \frac{t}{3}\right)>1-r
$$

$$
M\left(x, y, \frac{t}{3}\right)>1-r
$$

for all $x, y \in A_{n_{0}}$. Let $x, y \in F_{n_{0}}$. Then there exists sequences $\left\{x_{n}^{\prime}\right\}$ and $\left\{y_{n}^{\prime}\right\}$ in $A_{n_{0}}$ such that $x_{n}^{\prime}$ converges to $x$ and $y_{n}^{\prime}$ converges to $y$. Hence $x_{n}^{\prime} \in B\left(x, r, \frac{t}{3}\right)$ and $y_{n}^{\prime} \in$ $B\left(y, r, \frac{t}{3}\right)$ for sufficiently large $n$. Now

$$
\begin{gathered}
M(x, y, t) \geq M\left(x, x_{n}^{\prime}, \frac{t}{3}\right) * M\left(x_{n}^{\prime}, y_{n}^{\prime}, \frac{t}{3}\right) * M\left(y_{n}^{\prime}, y, \frac{t}{3}\right) \\
>(1-r) *(1-r) *(1-r) \\
>(1-s)
\end{gathered}
$$

Therefore

$$
M(x, y, t)>1-s
$$

for all $x, y \in F_{n_{0}}$. Thus $\left\{F_{n}\right\}$ has fuzzy diameter zero. Hence by hypothesis $\cap_{n=1}^{\infty} F_{n}$ is nonempty. Take

$$
x \in \cap_{n=1}^{\infty} F_{n} .
$$

Then for $r, t>0,0<r<1$, there exists $n_{1}$ such that

$$
M\left(x_{m}, x, t\right)>1-r
$$

for all $n \geq n_{1}$. Therefore, for each $t>0, M\left(x_{n}, x, t\right)$ converges to 1 as $n$ tends to $\infty$. Hence $\left\{x_{n}\right\}$ converges $x$. Therefore $(X, M, *)$ is a complete fuzzy metric space.

Conversely,

Suppose that $(X, M, *)$ is fuzzy complete and $\left\{F_{n}\right\}_{n=1}^{\infty}$ is a nested sequence of nonempty closed sets with fuzzy diameter zero. Let $x_{n} \in F_{n}, n=1,2,3, \ldots$ Since $\left\{F_{n}\right\}$ has a diameter zero, for $\quad r, t>0,0<r<1$, there exists $n_{0} \in N$ such that

$$
M(x, y, t)>1-r
$$

for all $x, y \in F_{n_{0}}$.

Therefore

for all $n, m \geq n_{0}$. Since

$$
M\left(x_{n}, x_{m}, \frac{t}{3}\right)>1-r
$$

$$
x_{n} \in F_{n} \subset F_{n_{0}}
$$




\section{International Journal of Science and Research (IJSR) \\ ISSN (Online): 2319-7064}

Index Copernicus Value (2013): 6.14 | Impact Factor (2014): 5.611

and

$$
x_{m} \in F_{m} \subset F_{n_{0}},
$$

$\left\{x_{n}\right\}$ is a Cauchy sequence. But $(X, M, *)$ is a complete fuzzy metric space and hence $\left\{x_{n}\right\}$ converges to $x$ for some $x \in X$. Now for each fixed $n, x_{k} \in F_{n}$ for all $k \geq n$.

Therefore

$$
x \in \overline{F_{n}}=F_{n}
$$

for every $n$, and hence $x \in \cap_{n=1}^{\infty} F_{n}$. This completes our proof.

Remark 1.2.5 The element $x \in \cap_{n=1}^{\infty} F_{n}$ is unique. For if there are two elements , $y \in \cap_{n=1}^{\infty} F_{n}$,

since $\left\{F_{n}\right\}_{n=1}^{\infty}$ has fuzzy diameter zero, for each fixed

$$
t>0, M(x, y, t)>1-\frac{1}{n},
$$

for each $n$. This implies

and hence

$$
M(x, y, t)=1
$$

$$
x=y \text {. }
$$

Here we start by providing an extension of Theorem 1.1.2 to the context of fuzzy metric spaces.

Theorem 1.2.6 Every separable fuzzy metric space is second countable.

Proof: Let $(X, M, *)$ be the given separable fuzzy metric space. Let $A=\left\{a_{n}: n \in \mathbb{N}\right\}$, be a countable dense subset of $X$. Consider

$$
B=\left\{B\left(a_{j}, \frac{1}{k}, \frac{1}{k}\right): j, k \in \mathbb{N}\right\} .
$$

Then $B$ is countable. We claim that $B$ is a base for the family of all open sets in $X$. Let $G$ be an arbitrary open set in $X$. Let $x \in G$, then there exists $r, t>0,0<r<1$, such that

$$
B(x, r, t) \subset G .
$$

Since $r \in(0,1)$, we can find an $s \in(0,1)$ such that

Choose $m \in \mathbb{N}$ such that

$$
(1-s) *(1-s)>(1-r) \text {. }
$$

$$
\frac{1}{m}<\min \left(s, \frac{t}{2}\right)
$$

Since $A$ is dense in $X$, there exists $a_{j} \in A$ such that

Now if $y \in B\left(a_{j}, \frac{1}{m}, \frac{1}{m}\right)$ then,

$$
a_{j} \in B\left(x, \frac{1}{m}, \frac{1}{m}\right) \text {. }
$$

$$
\begin{gathered}
M(x, y, t) \geq M\left(x, a_{j}, \frac{t}{2}\right) * M\left(y, a_{j}, \frac{t}{2}\right) \\
\geq M\left(x, a_{j}, \frac{1}{m}\right) * M\left(y, a_{j}, \frac{1}{m}\right) \\
\geq\left(1-\frac{1}{m}\right) *\left(1-\frac{1}{m}\right) \\
\geq(1-s) *(1-s) \\
>1-r .
\end{gathered}
$$

Thus $y \in B(x, r, t)$ and hence $B$ is a basis. Hence the result. The next proposition generalizes Proposition 1.1.2.

Proposition 1.2.7A subspace of a separable fuzzy metric space is separable.

Proof: Let $X$ be the given fuzzy metric space and $Y$ be a subspace of $X$. Let

$$
A=\left\{x_{n}, n \in \mathbb{N}\right\}
$$

be a countable dense subset of $X$. For arbitrary but fixed $n, k \in \mathbb{N}$, if there are points $x \in X$ such that

$$
M\left(x_{n}, x, \frac{1}{k}\right)>1-\frac{1}{k},
$$

choose one of them and denote it by $x_{n_{k}}$. Let $B=\left\{x_{n_{k}}, n, k \in \mathbb{N}\right\}$, then $B$ is countable. Now we claim that $Y \subset \bar{B}$. Let $y \in Y$. Given $r, t>0,0<r<1$, we can find $a, k \in \mathbb{N}$ such that

$$
\left(1-\frac{1}{k}\right) *\left(1-\frac{1}{k}\right)>1-r \text {. }
$$

Since $A$ is dense in $X$, there exists an $m \in \mathbb{N}$ such that $M\left(x_{m}, y, \frac{1}{k}\right)>1-\frac{1}{k}$.

But by definition of $B$, there exists $x_{m_{k}} \in A$ such that

Now

$$
M\left(x_{m_{k}}, x_{m}, \frac{1}{k}\right)>1-\frac{1}{k} \text {. }
$$

$$
\begin{gathered}
M\left(x_{m_{k}}, y, t\right) \geq M\left(x_{m_{k}}, x_{m}, \frac{t}{2}\right) * M\left(x_{m}, y, \frac{t}{2}\right) \\
\geq M\left(x_{m_{k}}, x_{m}, \frac{1}{k}\right) * M\left(x_{m}, y, \frac{1}{k}\right) \\
\geq\left(1-\frac{1}{k}\right) *\left(1-\frac{1}{k}\right) \\
=1-r .
\end{gathered}
$$

Thus $y \in \bar{B}$ and hence $Y$ is separable.

\section{References}

[1] GEORGE A., VEERAMANI P., On some results in fuzzy metric spaces, Fuzzy Sets and System, 64(1994) 395-399.

[2] KRAMOSIL O., MICHALEK J., Fuzzy metric and statistical metric spaces, Kybernetica, 11(1975) 326-334.

[3] EISENBERG M., Topology, New York: Holt, Rinehart and Winston, 1974.

[4] HAASER N.B., Real Analysis/Norman B. Haaser, Joseph A. Sullivan, New York: Dover Publication, 1991.

[5] KREYSIG E., Introductory functional Analysis with applications, New York: Wiley, 1978.

[6] LIPSCHUTZS S., Schaums outline of theory and problems of general topology, New York: Schaum, 1965.

[7] MUNKRES J.R., Topology: a first Course, Englewood Cliffs N. J.: Prentice Hall, 1974.

[8] SHIRALI S., Metric Spaces/Satish Shirali and Harkrishan Vasudera L., London: Springer, 2006. 